\title{
Conceitos Básicos de Estatística: as Lacunas Conceituais de Acadêmicos Ingressantes no Curso Superior
}

\author{
Basic Statistics Concepts: The Conceptual Gaps of Students \\ Entering Higher Education
}

Rejane Padilha Quedi ${ }^{1}$; Luiz Marcelo Darroz ${ }^{1}$

\begin{abstract}
RESUMO
Apresentam-se os resultados de uma investigação que buscou identificar as lacunas conceituais sobre conceitos básicos de estatística de acadêmicos ingressantes no curso superior. Participaram da pesquisa 200 acadêmicos de uma universidade do Norte do Rio Grande do Sul, que, na época da coleta de dados, ainda não haviam cursado disciplinas da graduação abordando conceitos de estatística. Como instrumento de coleta de dados, utilizou-se um questionário com quatro questões abertas sobre representação gráfica, dados em série e agrupados, que abordavam conceitos de média aritmética, moda, mediana, desvio padrão, interpretação tabular e gráfica. Para evidenciar as lacunas, analisaram-se os dados a partir do número de respostas certas e erradas. Ainda, para examinar se as respostas dos egressos de escolas da rede pública de ensino apresentam diferenças estatisticamente significativas em relação às dos acadêmicos que cursaram o ensino médio em instituições privadas de ensino, aplicou-se o teste exato de Fisher. Os resultados apontam a existência de lacunas conceituais entre os participantes para dados em série e dados agrupados. Os resultados também evidenciam que para alguns elementos pesquisados as respostas dos acadêmicos que cursaram o ensino médio em escolas públicas apresentam diferenças estatisticamente significativas em relação às dos egressos de escolas da rede privada.
\end{abstract}

Palavras-chave: Ensino de estatística. Lacunas conceituais. Educação básica.

\begin{abstract}
This work presents the results of an investigation that aimed to identify the conceptual gaps on basic statistics concepts of students entering higher education. Two hundred students from a university in northern Rio Grande do Sul, Brazil participated in the research; at the time of data collection, the students had not attended the course subjects addressing statistics concepts yet. The instrument for data collection was a questionnaire consisting of four open questions on graphic representation and serial and grouped data, which addressed the concepts of arithmetic mean, mode, median, standard deviation, and table and graphic interpretation. To evidence the gaps, the data were analyzed from the number of right and wrong answers given by the participants to the questions proposed. Additionally, Fisher's exact test was applied to examine whether the answers of alumni from public schools presented statistically significant differences relative to the ones provided by the students who attended high school in private educational institutions. The results indicate the existence of conceptual gaps among the students participating in the study, for both serial and grouped data. The results
\end{abstract}

${ }^{1}$ UPF - Universidade de Passo Fundo, Passo Fundo/RS - Brasil. 
also show that, for some elements investigated, the answers given by the students who attended high school in public schools present statistically significant differences relative to the answers of alumni from private schools.

Keywords: Statistics teaching. Conceptual gaps. Basic education.

\section{INTRODUÇÃO}

A sociedade contemporânea encontra-se em constante transformação, pois a todo instante, novas informações são divulgadas pelos diferentes meios de comunicação. Nesse contexto, a estatística é a ciência que vem se tornando uma importante ferramenta no auxílio da interpretação dessas informações (ECHEVESTE et al., 2002).

Dessa forma, o correto entendimento dos conceitos estatísticos e o desenvolvimento de competências relacionadas a essa área tornam-se fundamentais para as tomadas de decisões necessárias à compreensão do mundo moderno. Isto é, a realidade atual exige dos cidadãos a capacidade de sintetizar e analisar uma grande quantidade de informações, sendo indispensável para qualquer profissional o domínio de assuntos pertinentes ao seu cotidiano. Tal fato demanda a criação de estratégias e metodologias de ensino de estatística capazes de promover aprendizagens significativas, duradouras e profundas nos estudantes da educação básica. A esse respeito, Lopes (2010, p. 227) salienta que

[...] a presença constante da Estatística no mundo atual tornou-a uma realidade na vida dos cidadãos, levando-o à necessidade de ensinar estatística a um número de pessoas cada vez maior. Consequentemente, nas últimas décadas a maioria dos países introduziu, nos seus currículos de matemática, conteúdos de estatística, probabilidade e combinatória desde o início da escolaridade.

A recomendação de estudar conceitos básicos de estatística está presente, também, nos Parâmetros Curriculares Nacionais (PCN), nos Parâmetros Curriculares Nacionais do Ensino Médio (PCNEM) e nas Orientações Educacionais Complementares aos Parâmetros Curriculares Nacionais (PCN+). Tais documentos sugerem que o ensino da área deve ser introduzido nos anos iniciais, com a coleta de dados e a sua organização em tabelas e gráficos, resumindo-os e utilizando estatísticas de tendência central e dispersão, de forma a extrair padrões e tendências dos dados, com vistas à generalização.

De acordo com os PCN+ (BRASIL, 2002, p. 45),

[...] quando a escola promove uma condição de aprendizado em que há entusiasmo nos fazeres, paixão nos desafios, cooperação entre os partícipes, ética nos procedimentos, está construindo a cidadania em sua prática, dando as condições para a formação dos valores humanos fundamentais, que são centrais entre os objetivos da educação.

Porém, mesmo sendo evidente a necessidade de compreensão dos conceitos estatísticos, e embora os documentos oficiais recomendem o seu ensino durante a educação básica, percebe-se que, ao ingressarem no nível superior, os acadêmicos apresentam um alto índice de reprovação nas disciplinas que abordam esses conteúdos. Essa realidade revela a falta de compreensão e de domínio desses conceitos, seja na sua utilização em situações de interpretação ou na análise de dados (VENDRAMINI e DIAS, 2005). Diante disso, Woehlke e Leitner (1980) salientam ser necessária a realização de pesquisas sobre os processos de ensinar e aprender estatística para melhorar a compreensão do 
mundo contemporâneo e o desempenho de alunos de graduação e de pós-graduação nas disciplinas de seus respectivos cursos.

Frente a esse contexto, surgem as seguintes indagações: ao ingressarem num curso superior, os acadêmicos dominam os conceitos básicos de estatística? Esses sujeitos apresentam lacunas conceituais dos conteúdos da área? No caso da existência de lacunas conceituais, quais são elas? As dificuldades de estatística básica apresentadas por egressos de ensino médio de escolas da rede pública de ensino são equivalentes às de acadêmicos que cursaram essa etapa de ensino em escolas da rede privada? Na tentativa de responder a essas indagações, este trabalho apresenta os resultados de uma pesquisa que buscou identificar se há e quais são as lacunas conceituais sobre conceitos básicos de estatística de acadêmicos ingressantes no curso superior.

Pensa-se ser de fundamental importância conhecer o que os ingressantes de cursos superiores sabem sobre os conceitos básicos de estatística. Afinal, esse dado pode demonstrar as possíveis fraquezas do ensino da área em nível básico e, assim, chamar atenção dos docentes para uma nova abordagem dos conteúdos.

Para tanto, o trabalho estrutura-se da seguinte forma: na próxima seção, é apresentada uma reflexão sobre o ensino de estatística na atualidade; na terceira parte, descreve-se a metodologia da pesquisa; na sequência, apresentam-se e discutem-se os resultados alcançados; e no encerramento, expõemse as considerações finais.

\section{O ENSINO DE ESTATÍSTICA NA ATUALIDADE}

Esta seção tem por objetivo refletir sobre a importância da estatística e seu processo de ensino no contexto atual. Para isso, recorre-se a trabalhos contidos na literatura da área que podem servir de suporte para a investigação aqui apresentada.

De acordo com Echeveste et al. (2005), a estatística é o ramo da matemática responsável por métodos e técnicas de pesquisa envolvendo experimentos, coleta de dados, processamento, representações gráficas, análise e divulgação das informações. Para esses autores, apesar de ser uma ciência relativamente recente na área da pesquisa, a estatística remonta à antiguidade, quando operações de contagem populacional já eram utilizadas para obtenção de informações sobre os habitantes, riquezas e poderio militar dos povos. Segundo os autores,

O mais antigo dos registros estatísticos disponíveis foi o registro de presos egípcios, na data de 5000 a.C. Em 3000 a.C. há registros da falta de mão de obra relacionada à construção de pirâmides. Em 2238 a. C. o Imperador da China Yao fez o primeiro recenseamento com fins agrícolas e comerciais. No ano de 600 a. C. no Egito todas as pessoas declaravam todos os anos sua profissão e suas fontes de rendimento. $\mathrm{Na}$ Era de Cristo, ocorreu um recenseamento no qual as pessoas tinham que ser entrevistadas no local de sua origem (ECHEVESTE et al., 2005, p. 23).

$\mathrm{Na}$ atualidade, conforme Nogueira, Victer e Novikoff (2011), a estatística representa um poderoso instrumento para o conhecimento do mundo e domínio da natureza, pois reflete modos de analisar informações e de elaborar respostas a diferentes problemas do cotidiano. Assim, seus conceitos estão cada vez mais presentes no rol de conteúdos da educação básica, por ser compreendida como uma ciência que não se restringe a um conjunto de técnicas. Ao contrário, ela contribui com conhecimentos 
que permitem lidar com a incerteza e a variabilidade dos dados, mesmo durante a coleta, possibilitando tomadas de decisões com argumentos mais significativos (LOPES, 2003).

Nesse sentido, os PCN para a educação básica salientam que os conhecimentos da área devem fazer parte do cotidiano escolar, a fim de que o aluno consiga construir procedimentos para coletar, organizar, comunicar e interpretar dados, utilizando tabelas, gráficos e representações que aparecem frequentemente em seu dia a dia (BRASIL, 1997, p. 40). Essa característica da vida contemporânea traz ao currículo de Matemática uma demanda, a de abordar elementos da estatística, da combinatória e da probabilidade desde os ciclos iniciais (BRASIL, 1997, p. 84).

Bayer e Echeveste (2003), por sua vez, ressaltam que a evolução da importância da estatística e o debate sobre sua inserção nos currículos dos primeiros anos da escola resultaram na inclusão de seus conteúdos no programa da disciplina de Matemática. Porém, segundo os autores, muitos professores dessa disciplina, até mesmo os recém-formados, receberam poucos conhecimentos sobre a ciência na sua preparação profissional. A falta de preparação, muitas vezes, impede-os de trabalhar esses conteúdos em suas aulas, fato que, via de regra, origina lacunas conceituais nos estudantes da educação básica sobre os assuntos da área (BAYER; ECHEVESTE, 2003).

Confirmando o exposto, Quintas, Oliveira e Ferreira (2011) destacam que as principais lacunas conceituais enfrentadas por egressos da educação básica são originadas pela ausência da abordagem de conceitos estatísticos no ensino médio, como, por exemplo, aqueles ligados às propriedades das medidas de localização (moda, média e mediana) e à noção de desvio padrão. Tal concepção é compartilhada por Groth e Bergner (2005; 2006), ao afirmarem que os professores e estudantes apresentam as mesmas dificuldades cognitivas em relação aos conceitos estatísticos básicos, tais como amostra e medidas de tendência central. Segundo Cazorla et al. (1999), por sua vez, as pesquisas têm revelado que os estudantes demonstram dificuldades na utilização das ferramentas de análise de dados mais simples, citando a representação de dados em gráficos, para exemplificar.

No entendimento de Echeveste e Ávila (2002), essas dificuldades são preocupantes, uma vez que tais conhecimentos revelam-se básicos para a interpretação do mundo contemporâneo. As autoras os conceituam da seguinte forma:

Apresentação de dados: os dados obtidos em um estudo estatístico podem ser representados através de tabelas e/ou gráficos. As tabelas e os gráficos são extremamente úteis para sintetizar os valores que uma ou mais variáveis podem assumir, estes recursos permitem ao pesquisador demonstrar os resultados obtidos de uma forma muito mais clara e organizada;

Medidas de tendência central: as medidas de tendência central são valores numéricos que representam o centro de um conjunto de dados. O objetivo destas medidas é resumir, através de um único valor, todas as informações contidas em um grupo de dados de uma mesma variável;

Média Aritmética: é a medida de tendência central mais utilizada, sendo obtida pelo quociente da divisão da soma dos valores da variável pelo número de elementos do grupo de dados;

Mediana: de um conjunto de valores, ordenados por ordem de grandeza, é o valor situado de tal forma no conjunto que o separa em dois subconjuntos de mesmo número de elementos, ou seja, é o valor que ocupa a posição central em um conjunto de dados. Se o número de elementos da amostra for ímpar, a Mediana será exatamente o valor que se encontra na posição calcula. Já se tivermos um número 
par de observações, o valor da Mediana será a média dos dois valores centrais da distribuição;

Moda: é o valor que representa maior frequência no conjunto de dados, ou seja, o que mais se repete;

Medidas de Variabilidade: as medidas de variabilidade ou dispersão medem a variabilidade dos elementos em relação à sua média. É muito importante para o pesquisador saber qual a representatividade da média calculada para o conjunto de dados, ou seja, saber qual a variabilidade destes dados, se estes são homogêneos, ou não. Tão importante quanto representar um conjunto de dados através da média, é estudar a dispersão dos dados em torno da mesma. As medidas de variabilidade mais utilizadas são a variância e o desvio-padrão (ECHEVESTE; ÁVILA, 2002, p. 9294).

Para alterar esse cenário e promover um ensino de estatística que de fato contribua para a formação cidadã, é preciso possibilitar ao

aluno o confronto de problemas estatísticos com o mundo real, desafiando-os a encontrar soluções e estratégias para resolver os problemas que Ihes são apresentados. Cabe ao professor incentivar o aluno na busca e na socialização de estratégias, para que estes sejam capazes de ouvir as críticas e valorizar suas produções, bem como a de seus colegas, compreendendo que o aprendizado se dá na coletividade e o processo reflexivo enriquece o trabalho (SCHNEIDER; ANDREIS, 2013, p. 5).

Conforme Rumsey (1999), é difícil e consome tempo compor um trabalho estatístico que focalize os elementos pedagógicos básicos para que os estudantes possam fazer conexões, interpretar resultados, criar exemplos e pensar criticamente. Porém, o professor deve superar e eliminar suas próprias dificuldades para desenvolver esse importante trabalho. Bayer e Echeveste (2003) recomendam que um dos caminhos possíveis para minimizar essa situação seria identificar as dificuldades dos estudantes de nível médio. Isto é, para os autores, identificar as lacunas conceituais da área apresentadas pelos egressos da educação básica pode fornecer aos docentes de Matemática e/ou Estatística informações importantes para o redimensionamento dos estudos da área.

Diante desse contexto, buscou-se investigar quais são os conceitos básicos de estatística que correspondem a lacunas conceituais de ingressantes no curso superior.

\section{A PESQUISA}

O grupo de participantes desta investigação constituiu-se de 200 acadêmicos ingressantes no curso superior que, no segundo semestre de 2017, estavam matriculados nos cursos de Ciências Contábeis, Administração, Agronomia, Medicina Veterinária, Engenharia de Alimentos, Bacharelado em Química e Fabricação Mecânica de uma universidade comunitária do Norte do Rio Grande do Sul. Tendo em vista que o objetivo da pesquisa consistia em identificar as lacunas conceituais oriundas da educação básica, definiu-se que esses sujeitos não poderiam ter frequentado, até o momento da coleta de dados, disciplinas que apresentassem em seus conteúdos programáticos conceitos de estatística.

Como se pode perceber no gráfico da Figura 1, do total de participantes da pesquisa, 53,5\% eram do sexo masculino, 77\% haviam concluído o ensino médio na rede pública de ensino, e a maioria no ano de 2016. 
Figura 1 - Relação entre o número de pesquisados e o ano de conclusão do ensino médio

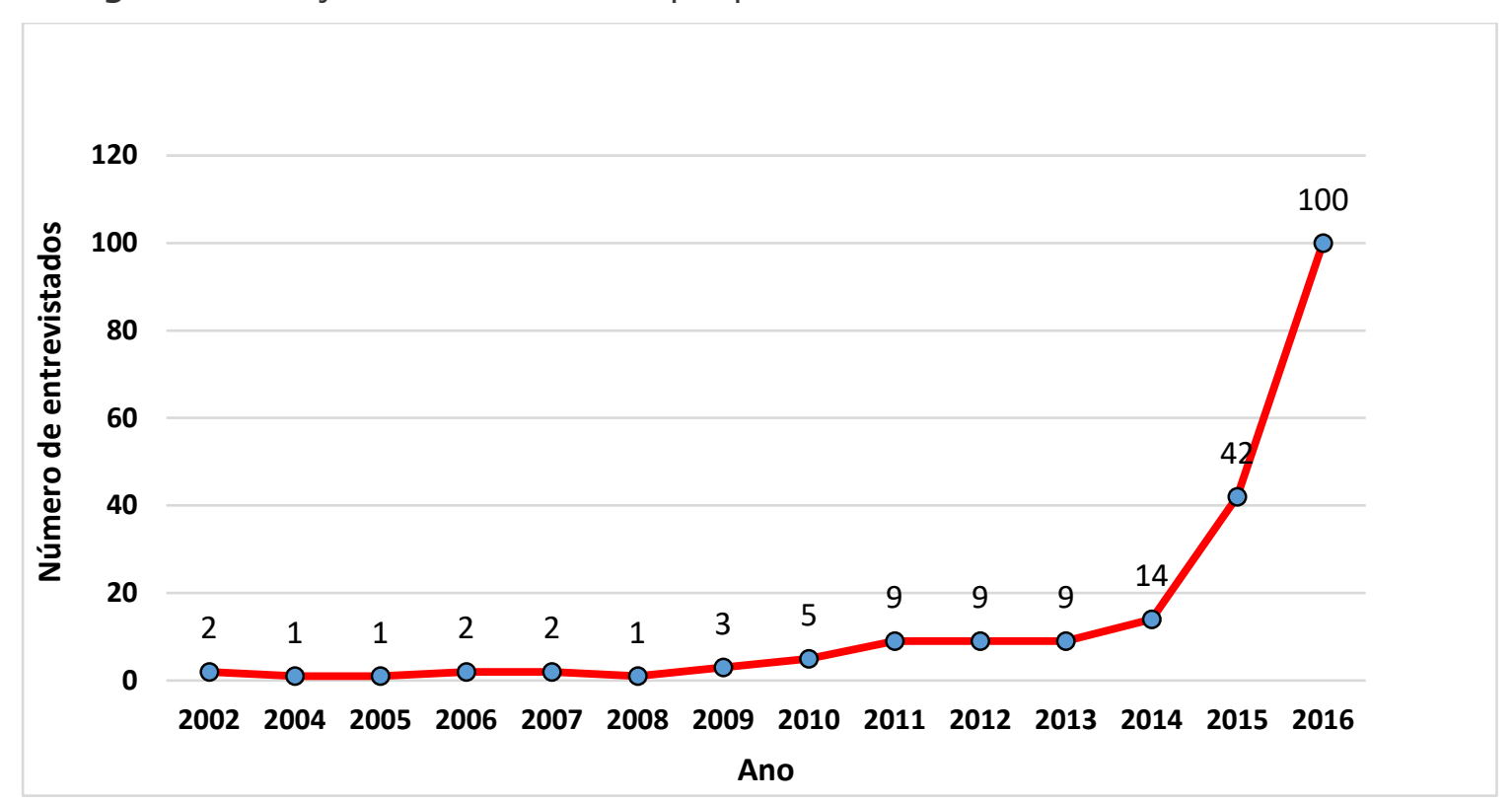

Fonte: dados da pesquisa, 2017.

Para a coleta dos dados, utilizou-se um instrumento de pesquisa (Anexo) composto por quatro questões abertas, elaborado especialmente para esta investigação. A primeira questão buscava avaliar a compreensão da análise gráfica dos acadêmicos para a determinação do cálculo da média aritmética. A segunda abordava dados em série com o intuito de verificar os conhecimentos sobre moda, mediana e desvio padrão. Na sequência, apresentava-se uma questão na qual os acadêmicos deveriam, com base na tabela, construir uma representação gráfica (linhas ou colunas). Por fim, a última questão solicitava que, a partir de uma representação tabular com dados agrupados, os participantes determinassem a média, a moda, a mediana e o desvio padrão para esses dados. Vale ressaltar que a opção por esses conceitos se deu com base nos trabalhos de Groth e Bergner (2006) e de Quintas, Oliveira e Ferreira (2011), apresentados na seção anterior e considerados os conceitos básicos sobre os quais os estudantes de nível médio evidenciam ter mais dificuldades.

A coleta de dados foi realizada na primeira aula do segundo semestre letivo de 2017 por professores da área de estatística. Os resultados, a serem discutidos na próxima seção, foram agrupados por índices de acertos de cada uma das questões e representados em gráficos para identificar as principais dificuldades enfrentadas pelos participantes e, dessa forma, perceber suas lacunas conceituais quanto aos conceitos básicos de estatística. Ainda, para identificar se existem diferenças estatisticamente significativas em relação às respostas dadas pelos acadêmicos, considerando a rede de ensino da qual são egressos - pública ou privada -, aplicou-se o teste exato de Fisher.

\section{RESULTADOS}

Após a correção das questões 1 e 2, os resultados foram agrupados de acordo com a rede de ensino onde os participantes cursaram o ensino médio e estão apresentados no gráfico a seguir (Figura 2). 
Figura 2 - Percentual de acertos relacionando as medidas para dados em série

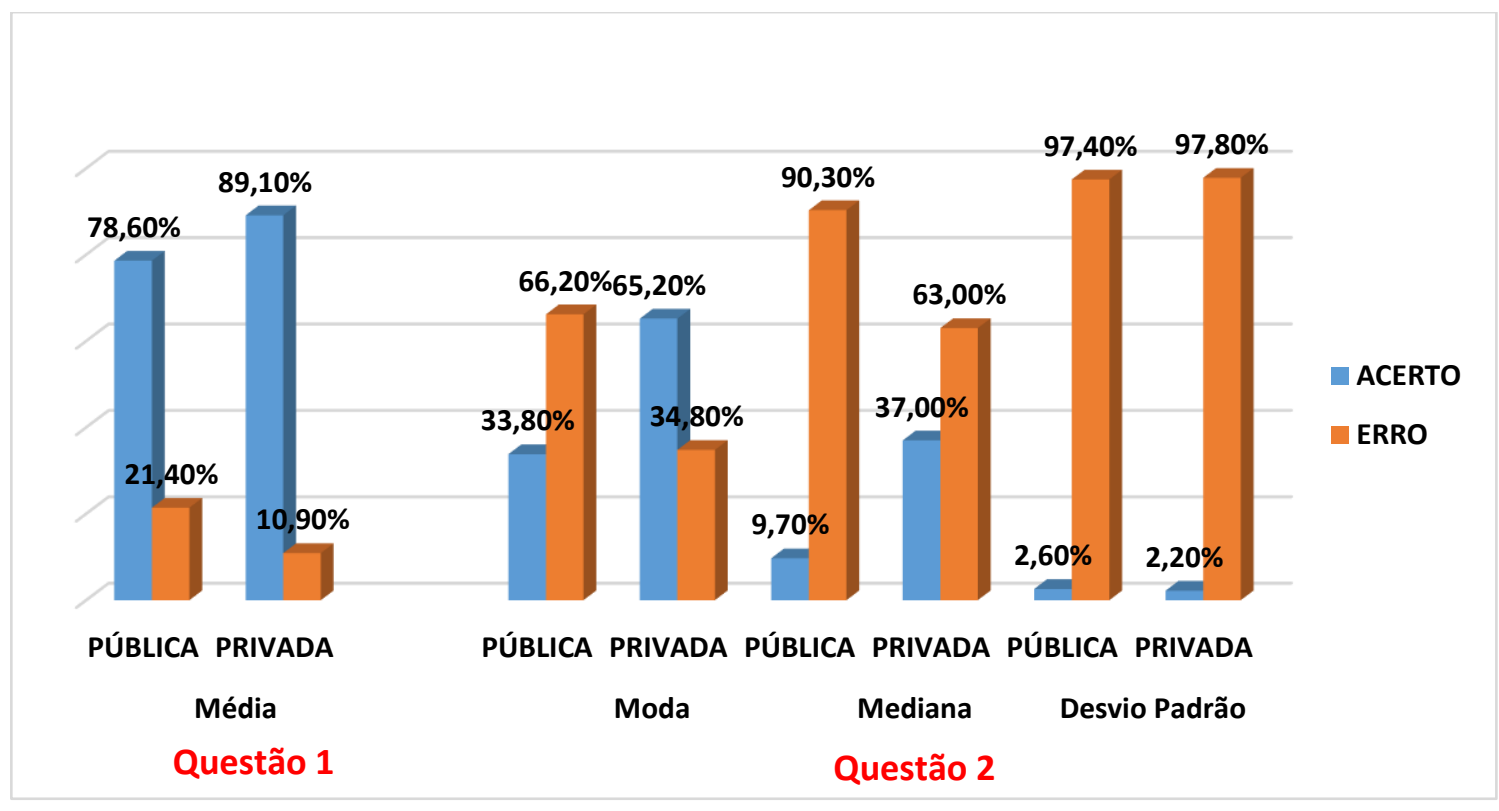

Fonte: dados da pesquisa, 2017.

Os dados contidos na Figura 2 demonstram que a maioria dos participantes da pesquisa (78,60\% dos egressos de escolas públicas e $89,10 \%$ dos egressos de escolas da rede privada) consegue retirar corretamente as informações de uma representação gráfica e realizar o cálculo da média aritmética. Esse fato indica que os conceitos estatísticos envolvidos são bem compreendidos pelos participantes, uma vez que muitos erraram a questão apenas devido a equívocos relacionados às operações de adição e multiplicação. Por esse motivo, conclui-se que retirar dados de uma representação gráfica e determinar a média aritmética de dados em série não corresponde a uma lacuna conceitual oriunda da educação básica.

Quanto à segunda questão, percebe-se, por meio dos dados contidos na Figura 2, que os conceitos de mediana e desvio padrão não são plenamente compreendidos pela maior parte dos participantes da pesquisa. Desses, o conceito de desvio padrão é o que apresenta o maior índice de erro, tendo em vista que a quase totalidade dos participantes, sejam oriundos de escolas públicas ou privadas, não relaciona esse tema a uma medida de dispersão em torno da média populacional de uma variável aleatória.

No que diz respeito à interpretação de mediana como o valor que separa a metade maior e a metade menor de uma amostra, uma população ou uma distribuição de probabilidade, nota-se que a maioria $(90,30 \%)$ dos acadêmicos que cursaram o ensino médio em escolas da rede pública apresentou dificuldades em determinar o conjunto de valores apresentados. Entre os participantes que realizaram seus estudos de nível médio em escolas privadas, o índice de respostas corretas aumenta. Porém, como se pode perceber nos dados do gráfico da Figura 2, a maioria $(63,00 \%)$ não conseguiu definir corretamente a mediana da série.

Os resultados relativos à moda, conceito que está relacionado às medidas de tendência central de um conjunto de dados, apresentam discrepâncias. Enquanto apenas 33,80\% dos participantes oriundos da rede pública de ensino conseguiram determinar a moda da série envolvida, 65,20\% dos egressos de instituições da rede privada de ensino acertaram o valor que corresponde à tendência central da série. 
Esses resultados apontam para a existência de dificuldades dos participantes egressos da rede pública de ensino nos três conceitos pesquisados. Os baixos índices de acertos e os erros cometidos na tentativa de resolver a questão permitem concluir que esses temas foram pouco ou não foram explorados no decorrer do período de nível médio nas redes públicas, de modo que esses conceitos parecem consistir em lacunas conceituais para esses sujeitos. Para os participantes que frequentaram escolas privadas, os resultados levam à conclusão de que uma das lacunas conceituais existentes se refere ao conceito de desvio padrão, embora a maioria desses egressos não tenha conseguido encontrar o valor correto para a mediana.

As respostas dadas à terceira questão, que solicitava que os participantes efetuassem uma representação gráfica dos dados apresentados, estão contidas na Figura 3.

Figura 3 - Percentual de acertos relacionando a representação gráfica

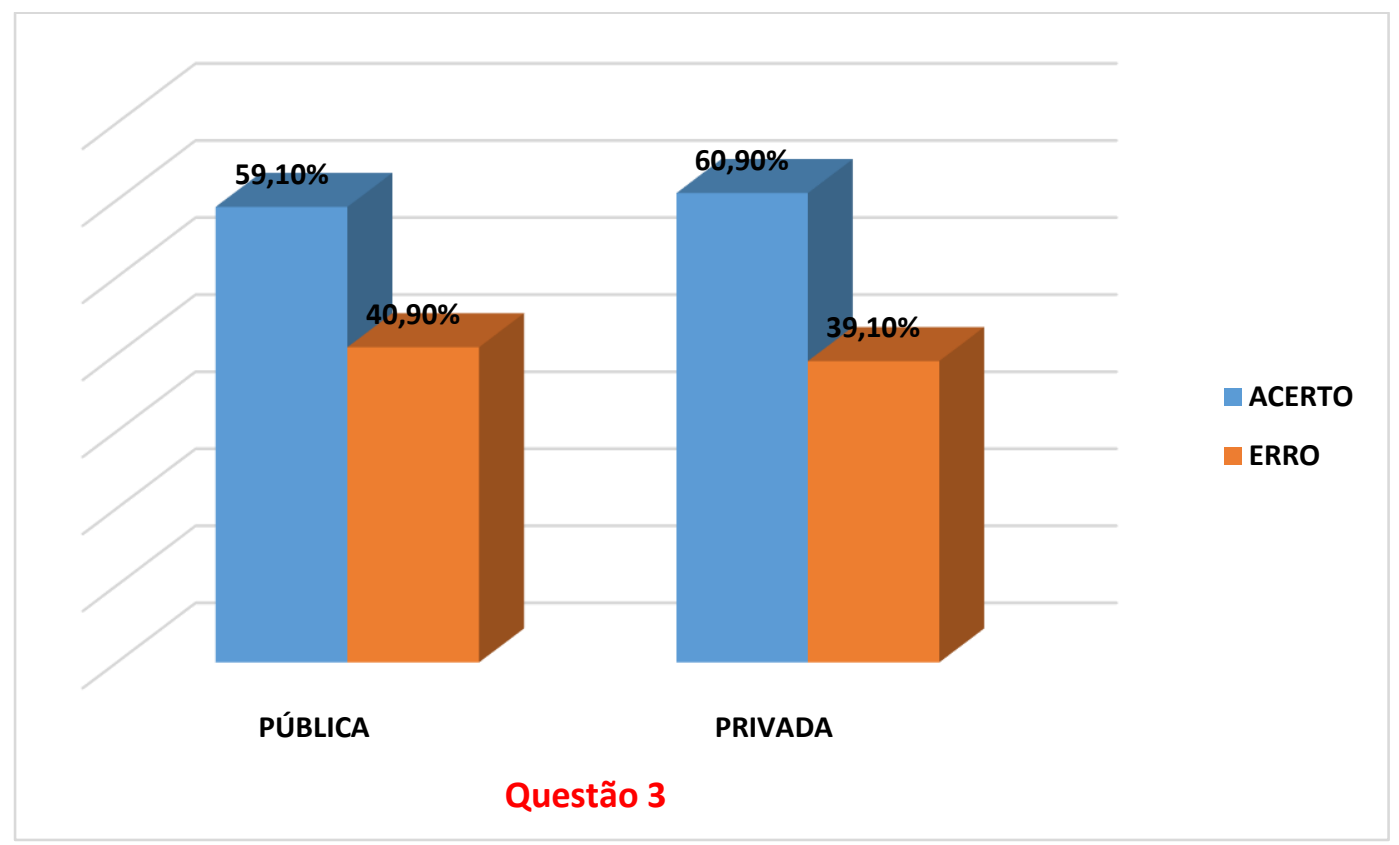

Fonte: dados da pesquisa, 2017.

Para essa questão, como mostra o gráfico da Figura 3, verifica-se que 59,10\% dos egressos de escolas públicas e 60,90\% dos egressos de escolas da rede privada conseguiram construir corretamente 0 gráfico a partir da representação tabular. Dessa forma, pode-se concluir que a maioria dos participantes não apresenta lacunas conceituais sobre o tema.

A última questão buscava identificar se os participantes, a partir de uma tabela com informações numéricas de tamanho grande, ou dados agrupados, conseguiriam determinar a média aritmética, a moda, a mediana e o desvio padrão. Após a correção, obtiveram-se os resultados apresentados no gráfico da Figura 4.

Conforme a Figura 4, a quarta questão mostrou-se uma tarefa difícil para todos os participantes. Os baixos índices de acerto evidenciados na efetivação do valor correspondente aos elementos básicos de estatística em foco demonstram que tanto os egressos do ensino público quanto os que frequentaram escolas da rede privada apresentam lacunas conceituais nesses assuntos. 
Figura 4 - Percentual de acertos relacionando as medidas para dados agrupados

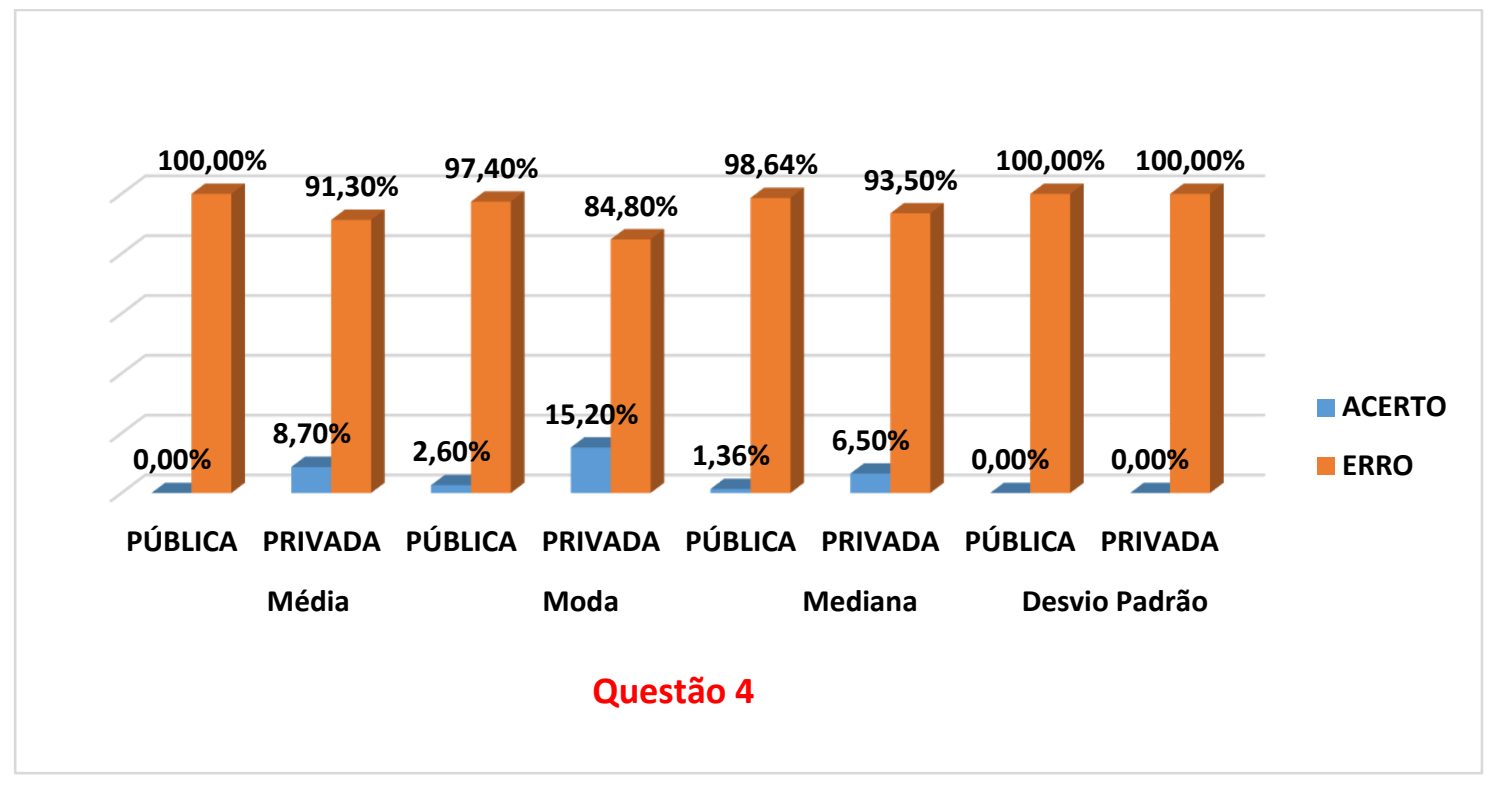

Fonte: dados da pesquisa, 2017.

Para ampliar a análise dos dados, aplicou-se o teste exato de Fischer, a fim de averiguar se as respostas dadas pelos acadêmicos que cursaram o ensino médio na rede pública apresentam diferenças estatisticamente significativas em relação às respostas dos egressos da rede privada. $A$ opção por este teste deve-se ao fato de que ele consiste em um teste de significância estatística utilizado na análise de tabelas de contingência $2 \times 2$, isto é, constitui-se de uma versão do teste quiquadrado. Ainda, seus resultados (valores de p) permitem rejeitar ou aceitar a hipótese inicial.

Os resultados obtidos para os conceitos de média aritmética, moda, mediana e desvio padrão para dados em série são apresentados no Quadro 1.

Quadro 1 - Análise do teste exato de Fisher para as medidas para dados em série

\begin{tabular}{|l|l|}
\hline Média aritmética & $p=0,0783$ \\
\hline Moda & $p=0,0001$ \\
\hline Mediana & $p=0,0001$ \\
\hline Desvio padrão & $p=0,6754$ \\
\hline
\end{tabular}

Fonte: dados da pesquisa, 2017.

Mediante a interpretação dos dados obtidos na determinação do cálculo da média aritmética ( $p=$ $0,0783)$ e do desvio padrão $(p=0,6754)$ dos dados em série, verifica-se que as respostas dos acadêmicos que cursaram o ensino médio em escolas da rede pública não apresentam diferenças estatisticamente significativas em relação às fornecidas pelos egressos de instituições privadas de ensino. Dessa forma, e considerando os índices anteriormente expostos, conclui-se que o conceito relativo à média aritmética é compreendido pelos participantes, enquanto os assuntos relacionados ao desvio padrão consistem em uma lacuna conceitual para a maioria deles, independentemente da rede de ensino frequentada no decorrer do ensino médio.

Para moda e mediana, os resultados do teste ( $p=0,0001$ para os dois conceitos) indicam que há diferenças estatisticamente significativas entre as respostas de egressos de escolas públicas e 
privadas. Os índices de acerto revelam que o desempenho dos egressos de escolas da rede privada é melhor que o dos ex-alunos de escolas públicas. Porém, na determinação da mediana, os índices de erro superam os de acerto nas respostas dadas pelos egressos de ambas as redes, levando a inferir que esse conceito também constitui uma lacuna conceitual para os dois grupos. Em relação à moda, os resultados apontam que o tema pode ser considerado uma lacuna conceitual para estudantes que cursaram o ensino médio na rede pública.

No que diz respeito à representação gráfica, solicitada na questão 3, o resultado do teste exato de Fisher $(p=0,4846)$ mostra não haver diferenças estatisticamente significativas entre o desempenho dos acadêmicos participantes em função da rede de ensino. Conforme os resultados anteriormente apresentados, os acadêmicos não parecem ter dificuldades quanto a esse assunto, conduzindo a concluir que essa não é uma lacuna conceitual na sua formação básica.

Os resultados do teste exato de Fischer para os dados agrupados estão contidos no Quadro 2. Ressalta-se que, nesse quadro, não está representado o resultado relativo à determinação do desvio padrão, uma vez que nenhuma das respostas dos participantes foi considerada correta.

Quadro 2 - Análise do teste exato de Fisher para as medidas para dados agrupados

\begin{tabular}{|l|l|}
\hline Média aritmética & $\mathrm{p}=0,0025$ \\
\hline Moda & $\mathrm{p}=0,0035$ \\
\hline Mediana & $\mathrm{p}=0,0810$ \\
\hline
\end{tabular}

Fonte: dados da pesquisa, 2017.

Segundo os resultados alcançados no teste exato de Fisher e apresentados no Quadro 2, evidenciase, através dos valores de significância, que, no cálculo da média aritmética e da moda para dados agrupados, existe diferença significativa entre os alunos de acordo com a rede de ensino frequentada, tendo melhor desempenho os egressos da rede privada. Para a mediana, verifica-se que não existe diferença significativa entre os dois grupos. No entanto, como já salientado, o baixo índice de acertos em ambos os estratos leva à conclusão de que esses três conceitos, acrescidos do tema desvio padrão para dados agrupados, correspondem a lacunas conceituais dos participantes da pesquisa.

\section{CONSIDERAÇÕES FINAIS}

Ramos (2007) destaca a estatística como uma ciência multidisciplinar que abrange praticamente todas as áreas do conhecimento humano. Para o autor, é através dela que se pode fazer análises e utilizar de resultados estatísticos na economia, na agronomia, na química, na física, na análise política, entre outros campos. Nesse sentido, a estatística tem sido utilizada para a otimização de recursos econômicos, aumento da qualidade e produtividade, para a análise de decisões políticas e judiciais e, principalmente, para a compreensão do mundo contemporâneo.

Por tudo isso, o domínio dos seus conceitos básicos é de fundamental importância na atualidade. Nessa perspectiva, este estudo buscou identificar as principais lacunas conceituais da área em um grupo composto por 200 acadêmicos ingressantes em cursos superiores de uma universidade do Norte do Rio Grande do Sul. 
De acordo com os resultados, ao ingressarem no curso de graduação, os estudantes não dominam os conceitos básicos de estatística, de modo que apresentam lacunas conceituais importantes na área, as quais podem representar dificuldades para o prosseguimento dos estudos em nível superior. A pesquisa identificou que essas lacunas residem, principalmente, nos dados agrupados. Nesse campo, percebeu-se que a maioria dos acadêmicos pesquisados não compreende como se determinam a média aritmética, a moda, a mediana e o desvio padrão. Para os dados em série, os resultados indicam a existência de lacunas conceituais, sobretudo, na determinação do desvio padrão e da mediana.

No que diz respeito às dificuldades de estatística básica apresentadas por egressos da rede pública de ensino e por aqueles que frequentaram a rede privada, o teste exato de Fisher demonstrou que, para alguns elementos pesquisados, existem diferenças estatisticamente significativas entre as respostas. Evidencia-se, claramente, que os acadêmicos que estudaram em escolas da rede privada apresentam melhor resultado nas respostas em relação aos egressos do ensino público. Tais constatações indicam que nos estabelecimentos públicos de ensino esses conceitos foram trabalhados superficialmente, ou não foram trabalhados.

Por fim, vale ressaltar que os resultados alcançados são oriundos de um campo limitado. Seria importante ampliar esta investigação para outras instituições de ensino superior e, dessa forma, identificar se as lacunas conceituais aqui evidenciadas estão presentes em outros contextos. No entanto, mesmo que o universo de abrangência seja restrito, a pesquisa anuncia uma situação problemática na educação básica. Apesar de todas as recomendações e orientações presentes na legislação vigente, os egressos dessa etapa de ensino ainda apresentam lacunas conceituais em elementos básicos de estatística.

\section{REFERÊNCIAS}

BAYER, Arno; ECHEVESTE, Simone. Estatística na escola: importância dos conteúdos de estatística no ensino fundamental e médio. Acta Scientiae, Canoas, v. 5, n. 1, p. 35-42, jan./jun. 2003.

BRASIL. Secretaria de Educação Fundamental. Parâmetros Curriculares Nacionais: Matemática (10 e $2^{\circ}$ ciclos do Ensino Fundamental). Brasília: MEC/SEF, 1997.

- Ministério da Educação. Secretaria de Educação Fundamental. Parâmetros Curriculares Nacionais: Matemática (30 e $4^{\circ}$ ciclos do ensino fundamental). Brasília: MEC, 1998.

Tecnologias. Brasília: MEC, 2002.

CAZORLA, Irene Mauricio; SILVA, Cláudia Borim da; VENDRAMINI, Claudette Maria Medeiros; BRITO, Márcia Regina Ferreira de. Adaptação e validação de uma escala de atitudes em relação à estatística. In: CONFERÊNCIA INTERNACIONAL: EXPERIÊNCIAS E EXPECTATIVAS DO ENSINO DE ESTATÍSTICA, 1, 1999, Florianópolis. Anais... Florianópolis: UFSC/ PRESTA/ IASE, p. 45-57, set. 1999.

ECHEVESTE, Simone; ÁVILA, Michele Gomes de. Estatística no Ensino Fundamental e Médio. Acta Scientiae, Canoas, v. 4, n. 1, p. 91-96, jan./jun. 2002.

ECHEVESTE, Simone; BITTENCOURT, Hélio; BAYER, Arno; ROCHA, Josy. Educação estatística: perspectivas e desafios. Acta Scientiae, Canoas, v. 7, n. 1, p. 103-109, jan./jun. 2005. 
GROTH, Randall; BERGNER, Jennifer. Pre-service elementary teachers' metaphors for the concept of statistical sample. Statistics Education Research Journal, v. 4, n. 2, p. 27-42, 2005.

LOPES, Celi Aparecida Espasandin. O conhecimento profissional dos professores e suas relações com a estatística e probabilidade na educação infantil. Campinas, SP: UNICAMP, 2003. Tese, Doutorado em Educação Matemática, Universidade Estadual de Campinas, 2003.

LOPES, Celi Aparecida Espasandin. (Org.). Estudos e reflexões em educação estatística. Campinas, SP: Mercado de Letras, 2010. p. 212-230.

NOGUEIRA, Paulo Apolinário; VICTER, Eline das Flores; NOVIKOFF, Cristina. Roteiro didático para o ensino de estatística: a cidadania na/pela matemática. Rio de Janeiro: Unigranrio, 2011. Disponível em: <https://bit.ly/2HKYDze>. Acesso em: 03 nov. 2017.

QUINTAS, Sandra Maria Oliveira; OLIVEIRA, Hélia Margarida Aparício Pintão de; FERREIRA, Rosa Antónia de Oliveira Figueiredo Tomás. O conhecimento didáctico em estatística: um estudo exploratório com professores de matemática do ensino secundário. Nuances: Estudos sobre Educação, v. 18, n. 19, p. 36-51, jan./abr. 2011.

RAMOS, Edson Marcos Leal Soares. Estatística: poderosa ciência ao alcance de todos. Jornal da Universidade Federal do Pará, ano XXX, n. 130, abr./maio 2016. Disponível em: <https://bit.ly/2KJ8SdF>. Acesso em: 03 nov. 2017.

RUMSEY, Deborah. Cooperative teaching, opportunities for introductory statistics teachers. The Mathematics Teacher, v. 92, n. 8, p. 734-737, nov. 1999.

SCHNEIDER, Juliana Cristina; ANDREIS, Rosemari Ferrari. Contribuições do ensino de estatística na formação cidadã do aluno da educação básica, 2013. Disponível em: <https://bit.ly/2ybmacU>. Acesso em: 03 nov. 2017.

VENDRAMINI, Claudette Maria Medeiros; DIAS, Anelise Silva. Teoria de Resposta ao Item na análise de uma prova de estatística em universitários. Psico-USF, Campinas, SP, v. 10, n. 2, p. 201-210, jul./dez. 2005.

WOEHLKE, Paula; LEITNER, Dennis. Gender differences in performance on variables related to achievement in graduate-level educational statistics. Psychological Reports, v. 47, p. 1119-1125, 1980.

Submetido: 09/07/2018

Aceito: $25 / 08 / 2018$ 


\section{ANEXO - Instrumento de coleta de dados aplicado na investigação}

\section{DADOS DE IDENTIFICACÃ̃O:}

- Curso:

- Em que ano você concluiu o ensino médio?

- Que tipo de escola frequentou? ( ) Pública

- Sexo: ( ) Masculino ( ) Feminino

\section{QUESTÕES:}

1. O gráfico abaixo apresenta as notas de Marcelo nas disciplinas de Matemática, História e Geografia, nos quatro bimestres de um ano letivo. Calcule a média final de Marcelo na disciplina de Matemática.

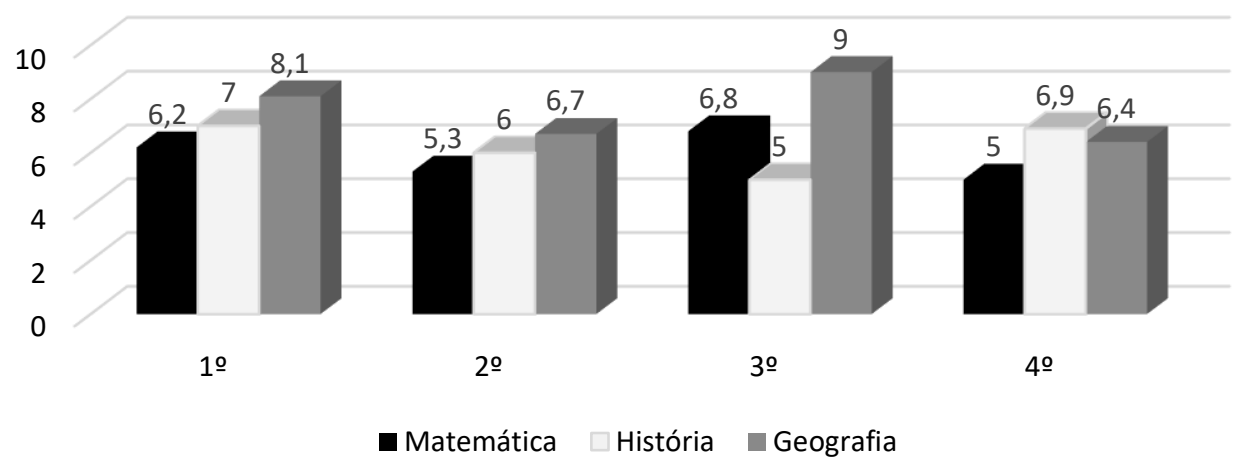

2. Determine a moda, a mediana e o desvio padrão do seguinte conjunto de valores:

$2,5,7,8,11,17,2,7,2$.

3. A partir da tabela abaixo, construa um gráfico de colunas ou de linhas:

Número de transplantes de pulmão no Brasil de 2008 a 2011

\begin{tabular}{|cc|}
\hline Ano & Número de transplantes \\
\hline 2008 & 53 \\
\hline 2009 & 59 \\
\hline 2010 & 61 \\
\hline 2011 & 49 \\
\hline
\end{tabular}

Fonte: <https://bit.ly/2LOvEd1>.

4. Observe a tabela e determine a média aritmética, a moda, a mediana e o desvio padrão da vida útil dos aparelhos.

Fabricação de aparelhos de MP3 - Indústria "XXX" - XXXXXXXXX/RS - Junho/2017.

\begin{tabular}{cc}
\hline $\begin{array}{c}\text { Vida útil } \\
\text { (meses) }\end{array}$ & Número de aparelhos \\
\hline 6 & 7 \\
12 & 22 \\
18 & 40 \\
24 & 31 \\
\hline
\end{tabular}

Fonte: fictícia 